\title{
ON GROUP RINGS OF FINITE METABELIAN GROUPS
}

\author{
G. KARPILOVSKY
}

(Received 12 February 1979)

Communicated by $\mathbf{H}$. Lausch

\begin{abstract}
It is proved that a finite metabelian group $G$ is determined by its group ring $K G$ where the ring $K$ satisfies the following conditions.

$\left(^{*}\right) K$ is an integral domain of characteristic 0 in which no prime dividing the order of $G$ is invertible.

(**) $Z / m Z$ is a homomorphic image of $K$ where $m=$ exponent of $G^{\prime}$.

It is also shown that al! groups of order $2^{n}, n \leqslant 7$ are determined by their integral group rings.

1980 Mathematics subject classification (Amer. Math. Soc.): primary 20 C 05; secondary 16 A 26.
\end{abstract}

The aim of this paper is to prove that a finite metabelian group $G$ is determined by its group ring $K G$ where the ring $K$ satisfies the following conditions

(*) $K$ is an integral domain of characteristic 0 in which no prime dividing the order of $G$ is invertible

(**) $Z / m Z$ is a homomorphic image of $K$ where $m=$ exponent of $G^{\prime}$

This theorem generalizes Whitcomb's result (Whitcomb (1968)) which states that a finite metabelian group is determined by its integral group ring and also a result of Sehgal (1970) who proved that a finite metabelian $p$-group is determined by its $p$-adic group ring. We present our theorem in a generalized form which covers (for the case $K=Z$ ) Obayashi's result (Obayashi (1970)) established by cohomological methods. We also prove that a 2 -group which is abelian-by-dihedral of order 8 is determined by its integral group ring. The corresponding result for the case of a 2-group which is elementary abelian-by-dihedral of order 8 was proved by Obayashi (1970). Finally, we show that all groups of order $2^{n}, n \leqslant 7$, are determined by their integral group rings. 
In what follows $K G$ denotes a group ring of a group $G$ over an associative ring $K$ with $1, I(K, G)$ denotes the augmentation ideal of $K G, K G=K H$ means that $H$ is a normalized group basis of $K G$. We shall often write $I(G)$ instead of $I(K, G)$ when the precise situation will be clear from the content. If $S$ is a subset of $K G$ and $\Lambda$ is an ideal of $K G$ then $S+\Lambda=\{s+\Lambda \mid s \in S\}$. Finally, $O_{p}$ (respectively $\left.Z_{(p)}\right)$ stands for the ring of $p$-adic integers (respectively $p$-integral rationals).

LEMMA 1. Let $G$ be an arbitrary group, $K$ an arbitrary ring with $1, M$ and $N$ arbitrary subgroups of $G$. Then the following equalities hold

$$
\begin{gathered}
I(M) \cdot I(N) \cap I(N)=I(M \cap N) \cdot I(N) . \\
I(G) \cdot I(N) \cap I(N)=I(N)^{2} . \\
G \cap I+I(G) \cdot I(N)=N \cap 1+I(N)^{2} .
\end{gathered}
$$

Proof. By taking $M=G$ we see that (1) $\Rightarrow(2)$. Since $G \cap 1+K G \cdot I(N)=N$ it follows that $G \cap 1+I(G) \cdot I(N)=N \cap 1+I(G) \cdot I(N)=N \cap 1+(I(G) \cdot I(N) \cap I(N))$ and therefore (2) $\Rightarrow(3)$. Let $T$ be a set of all coset representatives of $M$ with respect to $M \cap N$. If $m=t n, n \in M \cap N$, then for $n^{\prime} \in N$ we have

$$
(m-1)\left(n^{\prime}-1\right)=(t-1)(n-1)\left(n^{\prime}-1\right)+(t-1)\left(n^{\prime}-1\right)+(n-1)\left(n^{\prime}-1\right) .
$$

Since the first and the second summand belong to $(t-1) I(N)$ and since

$$
(n-1)\left(n^{\prime}-1\right) \in I(M \cap N) \cdot I(N), \quad(m-1)\left(n^{\prime}-1\right) \in I(M \cap N) \cdot I(N)+(t-1) \cdot I(N)
$$

then

Let

$$
I(M) \cdot I(N)=I(M \cap N) \cdot I(N)+\sum_{1 \neq t \in T}(t-1) I(N) .
$$

$$
\begin{aligned}
x=y+\left(t_{1}-1\right)\left[\alpha_{11}\left(n_{1}-1\right)+\ldots\right. & \left.+\alpha_{1 s}\left(n_{s}-1\right)\right]+\ldots+\left(t_{k}-1\right)\left[\alpha_{k 1}\left(n_{1}-1\right)\right. \\
& \left.+\ldots+\alpha_{k s}\left(n_{s}-1\right)\right],
\end{aligned}
$$

where

$$
y \in I(M \cap N) \cdot I(N), \quad t_{j} \in T, n_{i} \in N, \quad 1 \leqslant i \leqslant s, \quad 1 \leqslant j \leqslant k .
$$

If $x \in I(N)$ then

$$
z=\alpha_{11} t_{1}\left(n_{1}-1\right)+\ldots+\alpha_{1 s} t_{s} \quad\left(n_{s}-1\right)+\ldots+\alpha_{k s} t_{k}\left(n_{s}-1\right) \in I(N)
$$

and since all elements of $N$ have coefficient 0 in $z, z=0$.

But $\left\{t_{1}\left(n_{1}-1\right), \ldots, t_{s}\left(n_{s}-1\right)\right\}$ is a linearly independent set and therefore

$$
\alpha_{11}=\ldots=\alpha_{1 s}=\ldots=\alpha_{s s}=0 .
$$

Hence $x=y \in I(M \cap N) \cdot I(N)$, proving the lemma.

Let $A$ be an abelian group of exponent $n$ and let $K=Z / m Z$ where $m \equiv 0(\bmod n)$. As in the case $K=Z$ the formula

$$
f\left(\sum_{a \in A}\left(\alpha_{a} \cdot 1\right)(a-1)\right)=\prod_{a \in A} a^{\alpha_{a}}\left(\alpha_{a} \in Z\right)
$$


defines a homomorphism of $I(A)$ onto $A$ with kernel $I(A)^{2}$. From this follows: $A \cap 1+I(A)^{2}=1$ and $A \cong I(A) / I(A)^{2}$. Moreover, since

$$
f\left(\sum_{a \in A}\left(\alpha_{a} \cdot 1\right)(a-1)\right)=f\left(\prod_{a \in A} a^{\alpha_{\mathbf{a}}}-1\right)
$$

the following congruence holds

$$
\sum_{a \in A}\left(\alpha_{a} \cdot 1\right)(a-1) \equiv \prod_{a \in A} a^{\alpha_{n}}-1 \quad\left(\bmod I(A)^{2}\left(\alpha_{a} \in Z\right)\right) .
$$

In general if $G$ is a group and $n$ is the exponent of $G / G^{\prime}$ then

and

$$
G / G^{\prime} \cong I(K, G)_{i} I^{2}(K, G)
$$

where $K=Z / m Z$ and $m \equiv 0(\bmod n)$.

Lemma 2. Let $A$ be a subgroup $G$ and let $K=Z / m Z$ where

$$
m \equiv 0(\bmod n), \quad n=\text { exponent of } A / A^{\prime} .
$$

Then the following properties hold

$$
K G \cdot I(A) / I(G) \cdot I(A) \cong A / A^{\prime}
$$

and

(8) If $A$ is abelian and $x \equiv g(\bmod K G \cdot I(A))$ for some $g \in G$, then there exists a unique element $g_{x}=g a(a \in A)$ such that $x \equiv g_{x}(\bmod I(G) \cdot I(A))$.

Proof. It follows from $K G=K+I(G)$ that $K G \cdot I(A)=I(A)+I(G) \cdot I(A)$ and the application of (2) and (5) yields

$$
K G \cdot I(A) / I(G) \cdot I(A) \cong I(A) / I(A) \cap I(G) \cdot I(A)=I(A) / I(A)^{2} \cong A / A^{\prime},
$$

proving (7). Finally, $x \equiv g(\bmod K G \cdot I(A))$ implies $x \equiv g+t(\bmod I(G) \cdot I(A)$ for some $t=\sum_{s \in A}\left(\alpha_{s} \cdot 1\right)(s-1) \in I(A)$.

Therefore $x \equiv g+(a-1)=(1-g)(a-1)+g a=g_{x}(\bmod I(G) \cdot I(A))$ where

$$
a=\prod_{s \in A} s^{\alpha_{s}} \text {. }
$$

Since $G \cap 1+I(G) \cdot I(A)=A \cap 1+I(A)^{2}=1$ the element $g_{x}$ is unique, proving the lemma.

Let $\alpha \rightarrow \bar{\alpha}$ be a ring homomorphism from $K$ onto $\bar{K}$ with kernel $\Lambda$ and let $G$ be an arbitrary group. Then the mapping $\phi: K G \rightarrow \bar{K} G$ defined by

$$
\phi\left(\sum_{g} \alpha_{g} g\right)=\sum_{g} \bar{\alpha}_{g} g
$$

determines the epimorphism of rings $K G$ and $\bar{K} G$ with $\operatorname{Ker} \phi=\Lambda G$. 
LEMMA 3. Let $G$ be a group and let $K$ be $a$ ring. Then $K G=K H$ implies $\bar{K} G=\bar{K} \phi(H)$ and $\phi(H) \cong H$. Therefore if $A$ and $N$ (respectively $B$ and $T$ ) are subgroups of $G$ (respectively of $H$ ) such that

then

$$
K G \cdot I(A)=K G \cdot I(B) \text { and } N+K G \cdot I(A)=T+K G \cdot I(A)
$$

$\bar{K} G \cdot I(\bar{K}, A)=\bar{K} G \cdot I(\bar{K}, \phi(B)) \quad$ and $\quad N+\bar{K} G \cdot I(\bar{K}, A)=\phi(T)+\bar{K} G \cdot I(\bar{K}, A)$.

Proof. All we have to do is to prove that there exists an isomorphism $\lambda$ of group rings $\bar{K} H$ and $\bar{K} G$ such that $\lambda(H)=\phi(H)$. Clearly, $K G=K H$ implies $\Lambda G=\Lambda H$. Consider the mappings

$$
\bar{K} H \stackrel{\hat{\lambda}_{1}}{\longrightarrow} K H / \Lambda H=K G / \Lambda G \stackrel{\lambda_{2}}{\longrightarrow} \bar{K} G
$$

where

$$
\lambda_{1}\left(\sum_{h \in H} \bar{\alpha}_{h} \cdot h\right)=\sum_{h \in H} \alpha_{h} \cdot h+\Lambda H \quad \text { and } \quad \lambda_{2}\left(\sum \alpha_{g} g+\Lambda G\right)=\sum_{g \in G} \bar{\alpha}_{g} g .
$$

Then $\lambda_{1}$ and $\lambda_{2}$ are ring isomorphisms and therefore $\lambda_{2} \lambda_{1}$ is also a ring isomorphism. It is easy to see that $\lambda_{2} \lambda_{1}$ is also a $\bar{K}$-module isomorphism and that $\left(\lambda_{2} \lambda_{1}\right)(h)=\phi(h)$ for any $h \in H$, proving the lemma.

Let $R$ be the ring of algebraic integers of a number field and let $u=\sum_{g} \alpha_{g} g$ be a unit of finite order in $R G$. It is well known (Berman (1955)) that if $\alpha_{1} \neq 0$ then $u=\alpha_{1} \cdot 1$. From this follows that every central unit of finite order in $R G$ is trivial. The following extension of this result belongs to Saksonov (1971).

LEMMA 4. Let $K$ be an integral domain of characteristic 0 in which no prime dividing the order of $G$ is invertible. If $u^{m}=1$ where $u=\sum_{g} \alpha_{g} g \in K G$ and if $\alpha_{1} \neq 0$ then $u=\alpha_{1} \cdot 1$. In particular, all central units of finite order in $K G$ are trivial.

Proof. Consider the regular representation of the group ring $\Phi(\varepsilon) G$, where $\Phi$ is the quotient field of $K$ and $\varepsilon$ a primitive $m$ th root of unity. Then

$$
\operatorname{tr}(u)=\alpha_{1} \cdot|G|=\varepsilon_{1}+\ldots \varepsilon_{|G|}
$$

where $\varepsilon_{i}^{m}=1$ and $\varepsilon_{i} \in \Phi(\varepsilon), i=1,2, \ldots,|G|$. Therefore $\alpha_{1}=|G|^{-1}\left(\varepsilon_{1}+\ldots \varepsilon_{|G|}\right)$. All we have to do is to prove that $\alpha_{1}$ is an algebraic integer. By looking at the $\operatorname{tr}\left(u^{r}\right)$ where $(r, m)=1$ we see that the set $\left\{\beta_{1}=\alpha_{1}, \beta_{2}, \ldots, \beta_{t}\right\}$ of all $Q$-conjugates to $\alpha_{1}$, belongs to $K$ and therefore $Z\left[\beta_{1}, \ldots, \beta_{t}\right] \leqslant K$.

Suppose that $\alpha_{1}$ is not an algebraic integer. Then there exists an elementary symmetric function $f$ of $t$ variables such that $f\left(\beta_{1}, \ldots, \beta_{t}\right)$ is not a rational integer. On the other hand, for some $l \in Z$ and some $k, 1 \leqslant k \leqslant t, f\left(\beta_{1}, \ldots, \beta_{t}\right)=|G|^{-k} l$ and therefore $f\left(\beta_{1}, \ldots, \beta_{t}\right)=(a / b) \in Z\left[\beta_{1}, \ldots, \beta_{t}\right]$ where $a, b \in Z,(a, b)=1, b>1$ 
and every prime divisor $p$ of $b$ also divides $|G|$. This shows that $(a / p) \in Z\left[\beta_{1}, \ldots, \beta_{t}\right]$. Since $(a, p)=1$ there exist $c, d \in Z$ such that $a c+d p=1$ whence

$$
\frac{1}{p}=\frac{a c \pm d p}{\rho}=\frac{a}{p} \cdot c+d \in Z\left[\beta_{1}, \ldots, \beta_{\mathrm{l}}\right] \leqslant K,
$$

which is a contradiction. This proves the lemma.

COROLLARY. Let $N \Delta G$ and let $\pi: K G \rightarrow K(G / N)$ be the canonical homomorphism where $K$ is as in Lemma 4. If $K G=K H$ then

$$
K(G / N)=K \pi(H) \text { and } K G \cdot I(N)=K H \cdot I\left(N^{*}\right),
$$

where $N^{*}=H \cap 1+K G \cdot I(N)$.

Proof. It suffices to show that $\pi(H)$ is a group basis for $K(G / N)$. Indeed, in this case $\pi$ can be regarded as the extension of the epimorphism $H \rightarrow \pi(H)$ by $K$ linearity, hence $\operatorname{Ker} \pi=K G \cdot I(N)=K H \cdot I\left(N^{*}\right)$.

Let $\sum_{x \in H} \alpha_{x} \cdot x=0\left(\alpha_{x} \in K\right)$ and let $\alpha_{h} \neq 0$ for some $h \in \pi(H)$. Then

$$
\alpha_{h} \cdot h=-\sum_{x \neq h} \alpha_{x} \cdot x
$$

and therefore there exists $y \neq h$ such that the coefficient of 1 in $y h^{-1}$ is nonzero. But in this case $y h^{-1}=\alpha \cdot 1(\alpha \in K)$ and since $H$ is normalized, $y=h$-contradiction. This proves the corollary.

THEOREM. Let $A$ be an abelian normal subgroup of a finite group $G$ and let $K G=K H$ where the ring $K$ satisfies $\left({ }^{*}\right)$ and $\left({ }^{* *}\right)$ for $n=$ exponent of $A$. If $N / A$ (respectively $M / A^{*}$ where $A^{*}=H \cap 1+K G \cdot I(A)$ ) is the centre of $G / A$ (respectively $\left.H / A^{*}\right)$ then there exists an isomorphism of $M$ onto $N$ carrying $A^{*}$ onto $A$.

Proof. We shall prove even a more general result, namely if $N$ and $M$ are subgroups of $G$ and $H$ respectively such that

$$
N \geqslant A, \quad M \geqslant A^{*} \text { and } N+K G \cdot I(A)=M+K G \cdot I(A)
$$

then there exists an isomorphism of $M$ onto $N$ carrying $A^{*}$ onto $A$. It follows from the corollary of Lemma 4 that $K G \cdot I(A)=K G \cdot I\left(A^{*}\right)$ and hence $|A|=\left|A^{*}\right|$.

By applying Lemma 3 we may assume that $K=Z / m Z$ where $m$ is a multiple of both the exponent of $A$ and the exponent of $A^{*}$. Multiplying both sides of the equality $K G \cdot I(A)=K G \cdot I\left(A^{*}\right)$ by $I(G)=I(H)$ we obtain $I(G) \cdot I(A)=I(H) \cdot I\left(A^{*}\right)$. It follows from (7) that $A \cong A^{*} /\left(A^{*}\right)^{\prime}$. Since $|A|=\left|A^{*}\right|$ then $A \cong A^{*}$ and therefore the application of (3) and (6) yields $H \cap 1+I(H) \cdot I\left(A^{*}\right)=1$. Let

$$
\pi: K G \rightarrow K(G / A)
$$


be the canonical homomorphism. Since $N+K G \cdot I(A)=M+K G \cdot I(A)$ then

$$
\pi(N)=\pi(M)
$$

and therefore $|N / A|=\left|M / A^{*}\right|$. Hence $|M|=|N|$. The same equality also implies that for every $h \in M$ there exists $g \in N$ such that $h \equiv g(\bmod K G \cdot I(A))$. By (8) there exists a unique $g_{h} \in N$ such that $h \equiv g_{h}(\bmod I(G) \cdot I(A))$.

Therefore the mapping $f: h \rightarrow g_{h}$ defines a homomorphism of $M$ into $N$. Since $|M|=|N|$ and since $\operatorname{Ker} f \leqslant H \cap 1+I(H) \cdot I\left(A^{*}\right)=1, f$ is actually an isomorphism of $M$ onto $N$. Let $b \in A^{*}$. Then $b-1 \in \operatorname{Ker} \pi=K G \cdot I(A)=I(A)+I(G) \cdot I(A)$ and it follows from (4) that $b-1 \equiv a-1(\bmod I(G) \cdot I(A))$ for some $a \in A$. Hence $b \equiv a(\bmod I(G) \cdot I(A))$ and $f(b)=a$. Thus it remains only to prove that $N$ and $M$ satisfy $N+K G \cdot I(A)=M+K G \cdot I(A)$. It follows from the corollary of Lemma 4 that $K(G / A)=K \pi(H)$. Since $\pi(N)$ (respectively $\pi(M)$ ) is the centre of $G / A$ (respectively $H / A^{*}$ ) the application of Lemma 4 yields $\pi(N)=\pi(M)$. Hence $N+K G \cdot I(A)=M+K G \cdot I(A)$, proving the theorem.

COROLlaRY 1. Let $G$ be a finite metabelian group and let the ring $K$ satisfy $\left({ }^{*}\right)$ and $\left({ }^{* *}\right)$. Then $G$ is determined by its group ring $K G$.

Proof. Take $A=G^{\prime}$. Then $N=G$ and $M=H$. Now apply the theorem.

The following proposition was suggested to me by Dr K. R. Pearson.

COROLlary 2. Let $G$ be a finite metabelian group and let $K=S^{-1} Z$ be the ring of fractions of $Z$ with respect to $S$, where $S=\{a \in Z \mid(a,|G|)=1\}$. Then the group ring $K G$ determines $G$.

Proof. All we have to do is to check that $K$ satisfies $\left({ }^{*}\right)$ and $\left({ }^{* *}\right)$. It is obvious that $K$ satisfies $\left(^{*}\right)$ and that the mapping $S^{-1} Z \rightarrow Z / m Z$ defined by $(a / b) \rightarrow \bar{a}(\bar{b})^{-1}$ where $m=$ exponent of $G^{\prime}$ and $\bar{a}=a+m Z$ is a ring epimorphism, proving the corollary.

Berman and Rossa (1966) and Whitcomb (1968) gave an example of two group bases in $Z D_{4}$ which are not conjugate in $U\left(Z D_{4}\right)$ but are conjugate in $U\left(0_{2} D_{4}\right)$. (In fact they are conjugate in $U\left(Z_{(2)} D_{4}\right)$. On the other hand, Weller (1972) proved that there are only two nonconjugate classes of group bases in $U\left(Z D_{4}\right)$.

COROLLARY 3. Let $G$ be a 2-group with abelian normal subgroup $A$ such that $G / A \cong D_{4}$. Then $G$ is determined by its integral group ring.

Proof. Let $Z G=Z H$ and let $\pi: Z G \rightarrow Z(G / A)$ be the canonical homomorphism. Then $Z \pi(G)=Z \pi(H), 0_{2} \pi(G)=0_{2} \pi(H)$ and there exists a unit $u \in 0_{2} G$ such that 
$u^{-1} \pi(H) u=\pi(G)$. Since $0_{2} G$ is a local ring there exists a unit $t \in 0_{2} G$ such that $\pi(t)=u$ and therefore $\pi\left(t^{-1} H t\right)=\pi(G)$. Thus if $\tilde{H}=t^{-1} H t$ then $0_{2} G=0_{2} \tilde{H}$ and $G+\mathrm{O}_{2} G \cdot I(A)=\tilde{H}+0_{2} G \cdot I(A)$. It follows from the proof of the theorem that in this case $G \cong \widetilde{H}$, proving the corollary.

CoRollary 4. Let $|G|=2^{n}, n \leqslant 7$. Then the group $G$ is determined by its integral group ring.

Proof. Every group of order $2^{n} n \leqslant 6$, is metabelian and group of order $2^{7}$ has a normal abelian subgroup $A$ of index 8 (Miller, Blichfeldt and Dickson (1961)). Suppose that $G$ is not metabelian. It follows from Berman (1955) that we can restrict ourself to the case when $G / A \cong D_{4}$. Now apply Corollary 3 .

\section{References}

S. D. Berman (1955), 'On the equation $x^{m}=1$ in an integral group ring', Ukrain. Mat. Ž. 7, 253-261.

S. D. Berman and A. R. Rossa (1966), 'Integral group rings of finite and periodic groups', Algebra and Math. Logic, Izdat Kiev, Univ. Kiev, 44-53.

G. A. Miller, H. F. Blichfeldt and L. E. Dickson (1961), Theory and applications of finite groups (Dover Publications, New York).

T. Obayashi (1970), 'Integral group rings of finite groups', Osaka J. Math., 253-256.

A. I. Saksonov (1971), 'Group rings of finite groups I', Publ. Math. Debrecen, 18, 187-209.

S. K. Sehgal (1970), 'Isomorphism of p-adic group rings', J. Number Theory 2, 500-508.

W. R. Weller (1972), The units of the integral group ring $Z D_{4}$ (Thesis, Pennsylvania State University).

A. Whitcomb (1968), The group ring problem (PhD Thesis, University of Chicago).

Department of Mathematics

La Trobe University

Bundoora 3083

Australia 